BMJ Open Sport \& Exercise Medicine

\title{
Effect of a gluteal activation warm-up on explosive exercise performance
}

\author{
Matt Parr, ${ }^{1,2}$ Phil DB Price, ${ }^{1}$ Daniel J Cleather ${ }^{1}$
}

To cite: Parr M, Price PDB, Cleather DJ. Effect of a gluteal activation warm-up on explosive exercise performance. BMJ Open Sport Exerc Med 2017;3 e000245. doi:10.1136/ bmjsem-2017-000245

Accepted 14 March 2017

\section{(a) CrossMark}

${ }^{1}$ School of Sport, Health and Applied Sciences, St Mary's University, Twickenham, UK ${ }^{2}$ Department of Strength and Conditioning, Leicester Tigers (Leicester Football Club), Leicester, UK

Correspondence to Daniel J Cleather; daniel. cleather@stmarys.ac.uk

\section{ABSTRACT}

Objectives To evaluate the effect of a gluteal activation warm-up on the performance of an explosive exercise (the high hang pull (HHP)).

Methods Seventeen professional rugby union players performed one set of three HHPs (with $80 \%$ of their one repetition maximum load) following both a control and activation warm-up. Peak electrical activity of the gluteus maximus and medius was quantified using electromyography (EMG). In addition, the kinematics and kinetics of nine players was also recorded using force plate and motion capture technology. These data were analysed using a previously described musculoskeletal model of the right lower limb in order to provide estimates of the muscular force expressed during the movement.

Results The mean peak EMG activity of the gluteus maximus was significantly lower following the activation warm-up as compared with the control $(p<0.05$, effect size $d=0.30)$. There were no significant differences in the mean peak estimated forces in gluteus maximus and medius, the quadriceps or hamstrings $(p=0.053)$, although there was a trend towards increased force in gluteus maximus and hamstrings following the activation warm-up. There were no differences between the ground reaction forces following the two warm-ups.

Conclusion This study suggests that a gluteal activation warm-up may facilitate recruitment of the gluteal musculature by potentiating the glutes in such a way that a smaller neural drive evokes the same or greater force production during movement. This could in turn potentially improve movement quality.

\section{INTRODUCTION}

The purpose of a warm-up is to prepare the body for activity and in particular to promote optimal performance and decrease injury risk. One aspect of a modern warmup is often a battery of 'activation' exercises (often therapeutic exercises) which are thought to promote the recruitment of specific musculature. ${ }^{1}$ The rationale behind this is again twofold-improved activation of key musculature might improve both the kinematics of movement (reducing injury risk) and the ultimate performance outcome. One common candidate for such activation protocols is the gluteal musculature. This is in part because the glutes are

\section{What are the key findings?}

Seventeen elite rugby union players performed an Olympic weightlifting exercise after both a control and a gluteal activation warm-up.

- There were no differences in the ground reaction forces after the two warm-ups. There was a decrease in electromyography following the activation warm-up, but in contrast there were clear trends that were consistent with an increased recruitment of the glutes and hamstrings.

- These findings support the clinical practice of prescribing gluteal activation exercises to facilitate recruitment of the glutes during activity.

- In addition, this study supports the notion that the mechanism of this improved recruitment is through a potentiation of the glutes such that increased force is expressed for a given neural impulse.

one of the main contributors to force production in lower limb extension, ${ }^{2-4}$ and in part because weakness or altered activity of the glutes is sometimes implicated in a range of musculoskeletal complaints including lower back pain ${ }^{5}{ }^{6}$ and anterior knee pain. ${ }^{7-10}$

A number of previous groups have investigated the effect of therapeutic gluteal activation exercise on athletic performance both acutely ${ }^{11-14}$ and over a short training period. ${ }^{15}$ The results of this research have been equivocal however; some authors reported modest increases in performance outcome, ${ }^{11-13}$ whereas others found no difference. ${ }^{14}{ }^{15}$ One reason for these equivocal results is that the majority of the previous research has only quantified performance outcome (eg, height jumped, power output) and has not sought to evaluate changes in kinematics, electromyography (EMG) or muscular forces.

The purpose of this study was therefore to perform the first comprehensive investigation of the effect of a gluteal activation warm-up on subsequent explosive activity, incorporating measures of performance 
outcome, but also kinematics and electromyography (EMG). A unique aspect of the research was the incorporation of a state-of-the-art musculoskeletal model of the lower limb ${ }^{16}$ which permits the estimation of the actual muscle forces expressed during movement. We hypothesised that the gluteal activation warm-up would facilitate increased force expression in the glutes during movement.

\section{METHODS}

\section{Experimental approach}

Nine professional rugby players (FB group) performed a high hang pull (HHP) after both a control and an activation warm-up (cross-over design). The kinetics and kinematics of their movement was input into a musculoskeletal model $\left(\right.$ FreeBody ${ }^{16}$ ) to calculate estimates of the muscular forces during the movement while EMG was used to simultaneously quantify the electrical activity of the gluteus maximus and medius. An additional eight professional rugby players performed the same protocol but were monitored using EMG alone (thus giving a cohort of 17 players who were analysed using EMG; ALL group).

\section{Subject characteristics}

Seventeen elite male Premiership rugby union players took part in this study (previous research that has found significant differences in performance outcome after a gluteal activation warm-up had group sizes between 10 and 22 subjects ${ }^{11-13}$ ). There were no differences between the complete cohort and the subcohort who were analysed using FreeBody (table 1). The study was approved by the ethical review board of St Mary's University and all subjects gave informed written consent prior to testing.

\section{Procedure}

Subjects performed the trial on a day without any scheduled club training. On arrival, EMG electrodes and retroreflective markers were placed on the subjects (subjects wore tight fitting clothing). Markers and electrodes remained in situ until the completion of the final test. Following electrode and marker placement, the subjects completed the control warm-up shown in table 2. Next, the subjects had a 1 min rest period before performing a set of three HHPs using a load equal to $80 \%$ of their one repetition maximum (1RM; the players' 1RMs were calculated by the club's strength and conditioning coach based on their previous test scores). Subjects stood with their right foot centred on the force plate (figure 1A). Kinematic, kinetic and EMG data were collected simultaneously as described below.

Following the control test, subjects then rested for $20 \mathrm{~min}$. They then repeated an identical protocol as for the first test, except the control warm-up was replaced with the activation warm-up illustrated in table 2. Finally, the subjects had a further $20 \mathrm{~min}$ rest before completing maximum voluntary contraction (MVC) testing using previously established methods. ${ }^{17}$ (In brief, the subjects extended the hip maximally while lying prone to maximally contract gluteus maximus and abducted the hip maximally from a side lying position to contract gluteus medius. In both instances, manual resistance was provided by one of the investigators.)

\section{Instrumentation}

\section{Motion capture}

The positions of 18 retroreflective markers (attached with adhesive spray to the anatomical landmarks described in our previous work ${ }^{16}$ ) were recorded at $200 \mathrm{~Hz}$ using an 11 camera Vicon motion capture system (Vicon MX system, Vicon Motion Systems, Oxford, UK). The ground reaction force was recorded simultaneously at $1000 \mathrm{~Hz}$ using a Kistler force plate (Kistler Type 9286AA, Kistler Instrumente, Winterthur, Switzerland) and synchronised with the kinematic data using the Vicon system.

\section{Electromyography}

EMG data were recorded from the gluteus maximus and medius at $1000 \mathrm{~Hz}$ using a Biopac MP150 data acquisition system (BIOPAC Systems, California, USA). The EMG electrode sites were shaved and then cleaned with alcohol wipes. EMG electrodes were placed following the guidelines of the Surface ElectroMyoGraphy for the Non-Invasive Assessment of Muscles project (SENIAM project; www.seniam. org). In particular, the gluteus maximus markers were placed $2 \mathrm{~cm}$ apart halfway between the line from the second sacral vertebrae and the greater trochanter of the femur, and the gluteus medius markers were placed $2 \mathrm{~cm}$ apart and halfway along the line connecting the iliac crest to the greater trochanter.

\section{Data analysis}

Musculoskeletal modelling approach

We employed a publicly available musculoskeletal model of the lower limb (FreeBody; www.msksoftware. org.uk) in order to calculate estimates of the lower limb forces expressed during the HHP. The FreeBody model is described in great detail in a number of separate publications which catalogue its development, ${ }^{18-22}$ the public version used in this study ${ }^{16}$ and the validation and verification of the model, ${ }^{23} 24$ and so only a brief description of the analysis approach is provided here.

FreeBody represents the right lower limb as a three-dimensional linked chain of five rigid segments representing the foot, calf, thigh, patella and pelvis where the location and orientation of each segment 


\begin{tabular}{lll} 
Table 1 Subject characteristics \\
\hline & ALL group & FB group \\
\hline No. of subjects & 17 & 9 \\
Age (years) & $26.0( \pm 3.9)$ & $24.7( \pm 3.5)$ \\
Height $(\mathrm{m})$ & $1.868( \pm 0.067)$ & $1.856( \pm 0.070)$ \\
Body mass $(\mathrm{kg})$ & $103.3( \pm 10.4)$ & $101.8( \pm 9.6)$ \\
1RM HHP $(\mathrm{kg})$ & $109.1( \pm 10.7)$ & $109.4( \pm 9.7)$ \\
\hline
\end{tabular}

1RM, one repetition maximum; HHP, hang pull from the high hang.

are calculated from the motion capture data. The geometry of the musculoskeletal system is then calculated based on the posture of the model using data taken from the cadaver studies of Klein Horsman and colleagues. $^{25}$ The equations of motion of the lower limb are posed in the global coordinate system using the wrench and quaternion notation of Dumas and colleagues ${ }^{26}$ and are parameterised on a frame by frame basis using the musculoskeletal geometry, segment kinematics, segment anthropometry ${ }^{27}$ and the force plate data. For each frame, this yields a system of 22 equations of motion with 193 unknown variables (muscle, ligament and joint contact forces), that is, an indeterminate system for which there are generally many possible solutions. In order to solve the equations of motion, the solution space is first narrowed by applying constraints based on the physiology of the musculoskeletal system (eg, muscles can only pull not push). The most physiologically likely solution is then selected using an optimisation approach. Specifically, the solution which minimises the sum of the muscle stresses and normalised ligament forces raised to the third power (equation $1^{19}$ $28{ }^{29}$ ) is found using the fmincon function of MATLAB (V.2016b; Mathworks, Natick, Massachusetts, USA) for each frame individually.

$$
\min _{F_{i}, L_{j}} J=\sum_{i=1}^{163}\left(\frac{F_{i}}{\text { Fmax }_{i}}\right)^{3}+\sum_{j=1}^{14}\left(\frac{L_{i}}{\operatorname{Lmax}_{i}}\right)^{3}
$$

where $F_{i}$ is the predicted force in the ith muscle; $\mathrm{Fmax}_{\mathrm{i}}$ is the maximum force capability of the ith muscle; $\mathrm{L}_{\mathrm{i}}$ is the predicted force in the ith ligament; $\operatorname{Lmax}_{i}$ is the failure limit of the ith ligament.

\section{EMG analysis}

EMG amplitude data were collected, rectified and smoothed to an epoch of $50 \mathrm{~ms}$ via the average over samples algorithm ${ }^{30} 31$ using the Acqknowledge data acquisition and analysis software (BIOPAC Systems, 42 Aero Camino Goleta, CA 93117, USA). The smoothed EMG data were then normalised against the MVCs.

\section{Statistical analysis}

The performance of each repetition of the HHP was normalised by reference to the position of the marker on the right anterior iliac spine-times $t_{0}=0$ and $t_{1}=1$ were defined to be when the vertical displacement of the marker was at its smallest and greatest, respectively. The normalised values were then interpolated using the spline function of MATLAB to find values at regular intervals of 0.01 between $\mathrm{t}=-1.0$ and $\mathrm{t}=1.02$. These values were then combined to produce mean composite curves for each time series, for each subject and each trial and then for the overall means for control and activation trials.

Peak values of the ground reaction force, muscle force estimates and joint angles were identified from the mean curves of each subject and differences between warm-ups were assessed with a multivariate repeated measures analysis of variance (ANOVA) in IBM SPSS Statistics V.22.0 (International Business Machines Corporation; alpha set to $\mathrm{p}<0.05$ a

Table 2 Control and activation warm-ups

\begin{tabular}{lll}
\hline Exercise & Control warm-up & Activation warm-up \\
\hline Stationary bike & 3 min & 3 min \\
\hline Inch worm & 2 sets of 8 & 1 set of 6 \\
\hline Bodyweight squat & 2 sets of 8 & 1 set of 6 \\
\hline Leg swing & 2 sets of 5 each leg & 1 set of 6 each leg \\
\hline Lunge & 2 sets of 4 each leg & - \\
\hline Press up & 2 sets of 8 & - \\
\hline Prone plank with hip extension (figure 1B) & - & 1 set of 6 each leg* \\
\hline Side plank with hip extension & - & 1 set of 6 each leg* \\
\hline Single leg squat & - & 1 set of 3 each leg \\
\hline
\end{tabular}

$\star$ The planks involved a $2 \mathrm{~s}$ hold of position at the top for each repetition. 

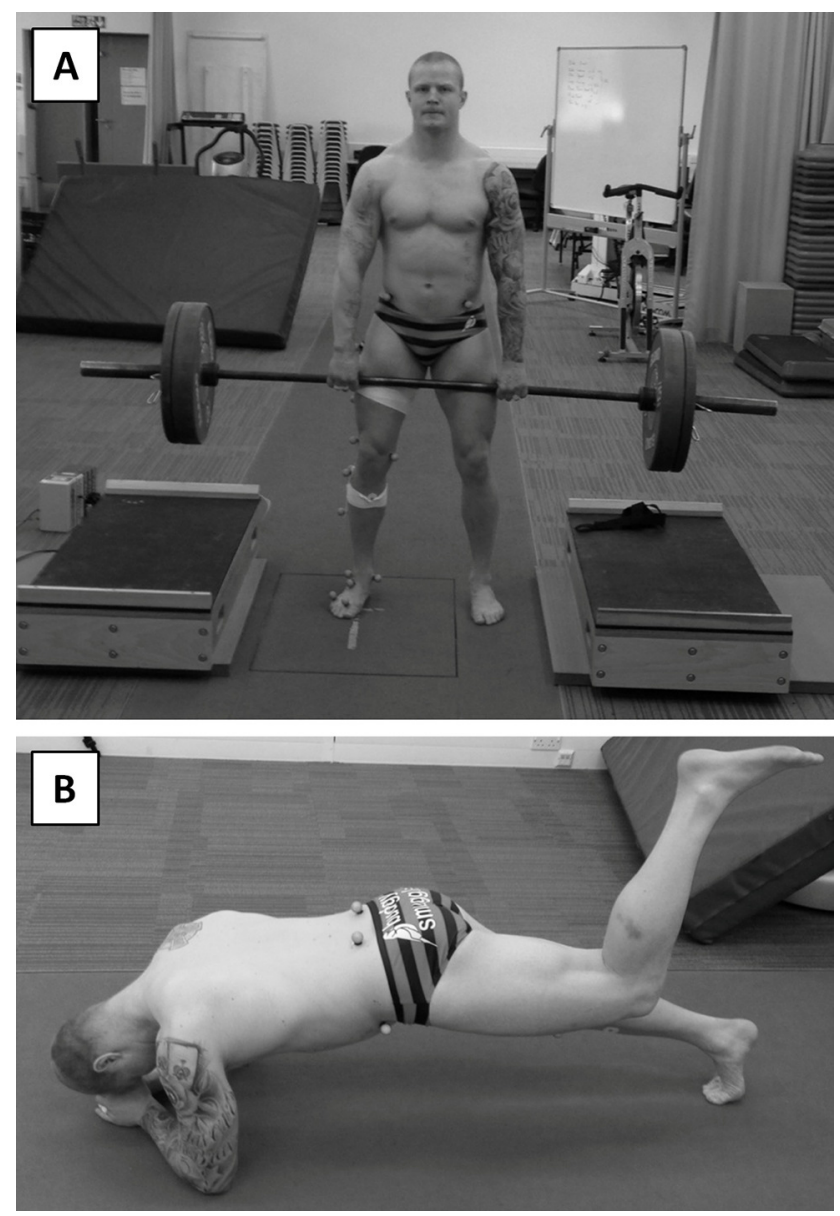

Figure 1 Illustrative images of a typical subject during a testing session. (A) Subject immediately prior to performing a high hang pull and (B) subject performing an activation exercise (prone plank with hip extension).

priori). The mean peak values of the normalised EMG signals and the baseline EMG signals (ie, the signal when the subject was holding the bar prior to the HHP) were found for each subject and trial. A two-factor repeated measures ANOVA was used to test for differences in this data $(\mathrm{p}<0.05)$. Finally, Cohen's d was calculated as a measure of effect size.

\section{RESULTS}

There were no differences in the ground reaction forces between control and activation trials (figure 2). Similarly, there were no statistically significant differences in hip or knee joint angles (figure 3), although the effect sizes of some of the differences in the peak non-sagittal plane angles were moderate to moderately large. In particular, during the activation trial, subjects' hips were more externally rotated $(\mathrm{d}=0.75, \mathrm{p}=0.12)$, whereas their knees were in a less varus position $(\mathrm{d}=0.54, \mathrm{p}=0.15)$.

The estimated muscle forces for the two trials are depicted in figure 2. There were no significant differences in the peak estimated muscle forces between the two trials $(p=0.053)$. The effect sizes of the increase in peak hamstring $(\mathrm{d}=0.68, \quad \mathrm{p}=0.07)$ and gluteus maximus forces $(\mathrm{d}=0.76, \mathrm{p}=0.05)$ were moderately large, whereas the effect size of the increase in peak gluteus medius $(\mathrm{d}=0.46, \quad \mathrm{p}=0.26)$ and quadriceps $(d=0.12, p=0.81)$ forces were smaller.

There were no statistically significant differences in the baseline EMG activity of either gluteus maximus or medius (figure 4). There was a trend for the mean peak EMG activity during the HHP to decrease from the control to the activation trial. This decrease was statistically significant for gluteus maximus when the cohort was considered as a whole (effect sizes of $\mathrm{d}=0.30$ and $\mathrm{d}=0.20$ for ALL and FB, respectively) and for gluteus medius when considering just the group that was analysed using FreeBody (effect sizes of $\mathrm{d}=0.28$ and $\mathrm{d}=0.49$ for ALL and FB, respectively).

\section{DISCUSSION}

\section{The effect of gluteal activation warm-up on performance of the HHP}

In this study, we sought to explore the effect of a gluteal activation warm-up on the performance of an explosive exercise (the HHP). The major findings of our study are as follows. First, there was no effect of the activation warm-up on the performance outcome (ie, there were no differences in the ground reaction forces). Second, there were no statistically significant differences in the kinematics of the HHP between the two warm-ups and the sagittal plane kinematics were markedly similar. However, the effect sizes of the peak differences in knee varus and external hip rotation were moderate and moderately large, respectively. Third, there were no significant differences in the peak estimated muscle forces; however, there was a trend for increased hamstring and gluteus maximus forces after the activation warm-up and the effect sizes of the differences in peak hamstring and gluteus maximus forces were moderately large. Finally, there were some statistically significant decreases in the EMG of the gluteal musculature after the activation warm-up of small to moderate effect size.

The clinical premise for performing gluteal activation exercises as part of a warm-up is that this will facilitate the use of the gluteal muscle group during activity. Despite the relatively small number of statistically significant differences found in this study, this research does tend to support this premise. In particular, the trends found among the muscle force estimates and the non-sagittal plane kinematics are consistent with the common clinical understanding of the impact of greater gluteal activation. That is, there was a greater external rotation of the hip that was commensurate with an increased force production by the glutes and that the knee was closer to a neutral alignment. In addition, the differences in muscle force estimates did approach significance $(p=0.053)$, and the effect sizes of the key differences in muscle force estimates and non- 


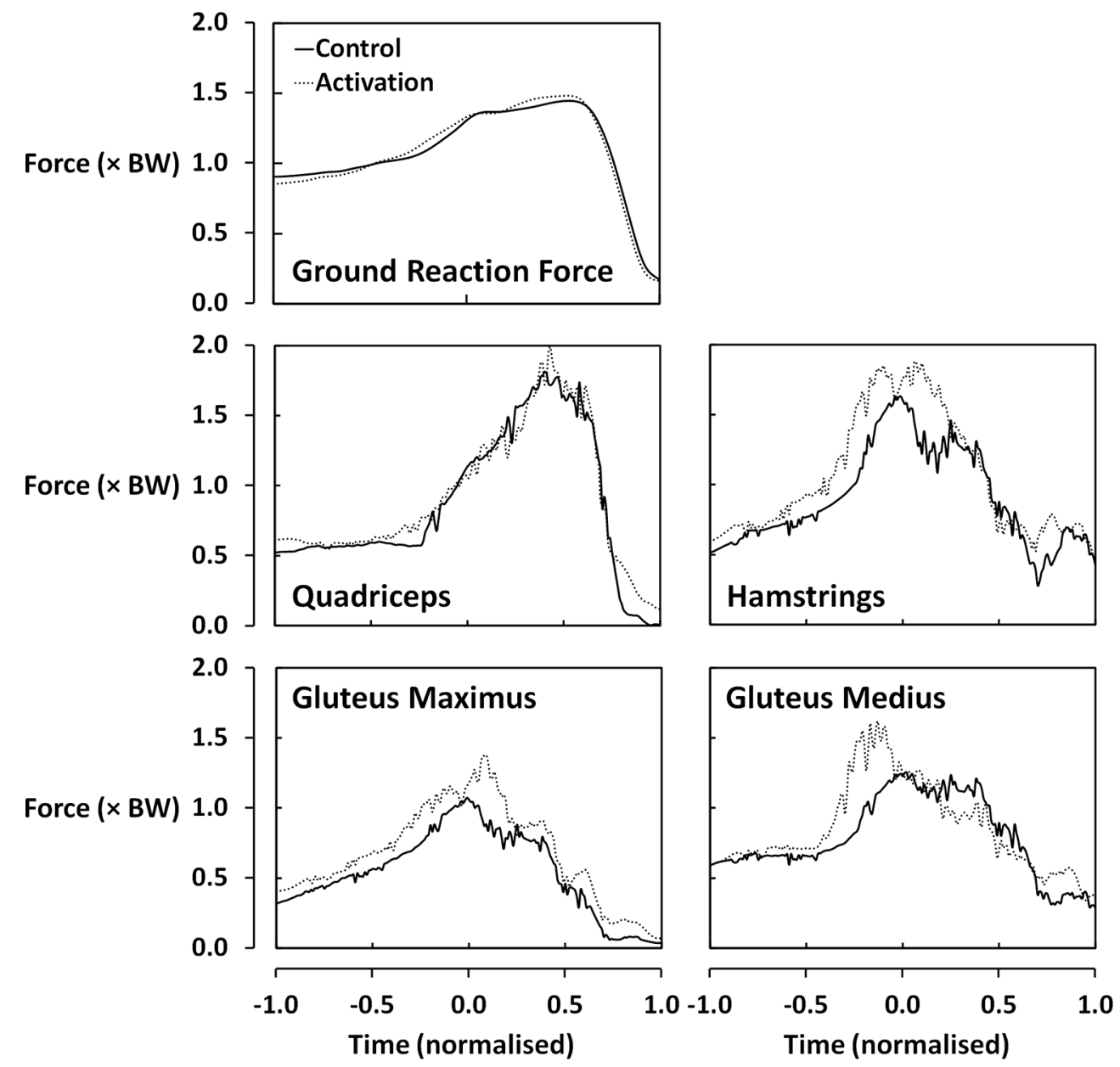

Figure 2 Ground reaction forces and estimated muscle forces during performance of a high hang pull after both a control and an activation warm-up (as a multiple of body weight (BW)).

sagittal plane kinematics were moderately large. Taken as a whole, these results do tend to suggest that a gluteal activation warm-up can change the relative muscular involvement within an activity and that this can have a positive impact on the posture of the lower limb.

In contrast with some of the previous literature, ${ }^{11-}$ 13 this study did not demonstrate any change in the performance outcome after activation warm-up. One reason for this may be that previous authors have been somewhat overeager to support the efficacy (and use) of gluteal activation warm-ups and have overstated the meaning of their results. For instance, Crow and colleagues ${ }^{11}$ argued that explosive power output was enhanced by an activation warm-up based on a small (effect size $=0.233$ ) but statistically significant increase in peak power output. This is especially bold given that an increase in peak power output does not necessarily mean there was an increase in jump height (jump heights were not reported). Similarly, Comyns and colleagues $^{12}$ reported that a gluteal warm-up can enhance force production based on changes in the ground reaction force-time curve, despite the fact that jump performance (height) was impaired for all of their post-warm-up jumps. The same group also suggested that a gluteal activation protocol can improve acceleration performance ${ }^{13}$ but again this was based on a small, significant effect size (a difference in $10 \mathrm{~m}$ sprint time of $0.02 \mathrm{~s} ; \mathrm{d} \approx 0.2$, $p=0.021)$. What is particularly surprising in all of this previous literature is the focus on investigating whether the performance outcome is improved, especially when the clinical rationale for including gluteal activation exercises in an athlete's programme is often more focused around improving movement quality.

Potentiation of the gluteal musculature by activation warmup?

One of the most interesting findings of this study was the fact that there was a significant decrease in the EMG signal following the activation warm-up, despite the fact that the ground reaction forces were unchanged and that there was a trend for the estimated muscle forces to increase. There are two candidates that might explain this finding. The first is that after the gluteal activation warm-up the 


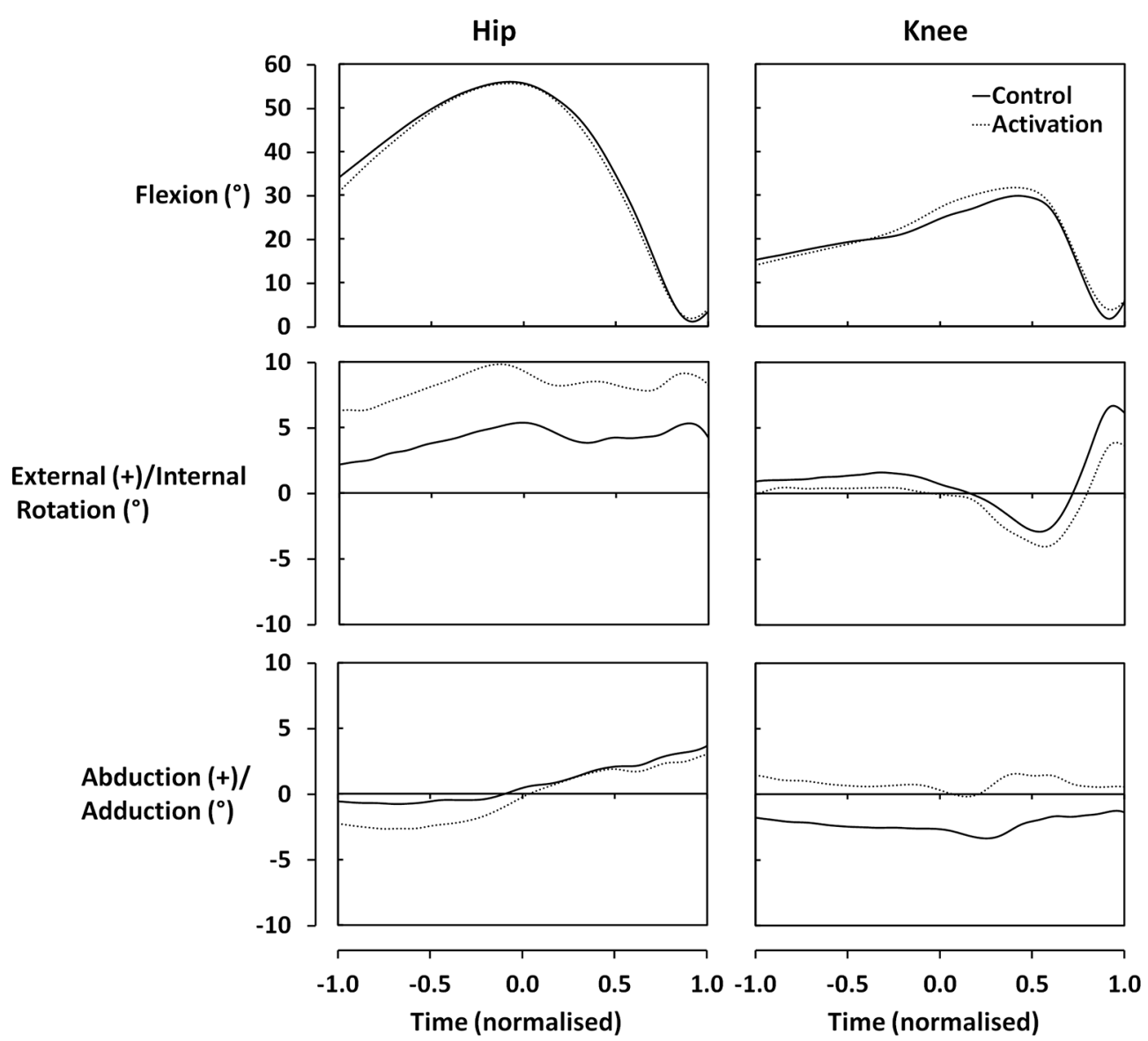

Figure 3 Hip and knee joint angles during the performance of a high hang pull after both a control and an activation warm-up.

kinematics of movement were altered in such a way that the glutes were able to operate at a more optimal position on their length-tension curve. This might then mean that a given level of neural drive would result in greater force production by the muscle. Certainly, our results did indicate that there may be some difference in the kinematics of the hip joint after the activation warm-up, but although the effect sizes of these differences were moderately large, they still only amounted to a few degrees, making this explanation seem less likely. The second possible explanation is that the gluteal activation

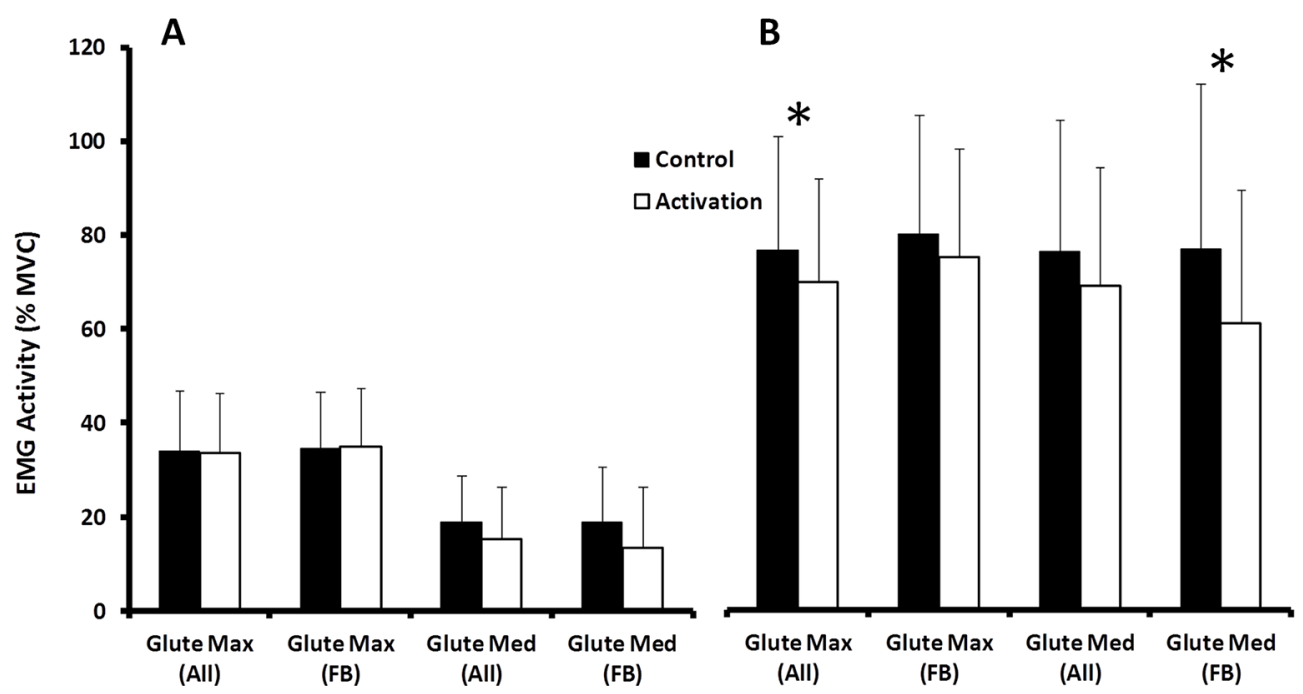

Figure 4 Mean peak electromyography (EMG) activity (\% maximum voluntary contraction (MVC)) of gluteus maximus and medius while holding the barbell $(\mathrm{A}$; baseline) and during the performance of a high hang pull $(\mathrm{B})$ after both a control and an activation warm-up (* indicates a significant difference between control and activation trials; $p<0.05$ ). 
warm-up potentiated the musculature of the glutes in such a way that the muscle contracted more strongly in response to a given neural signal. There is recent evidence $^{32}$ that specific training of the gluteal musculature can increase corticomotor excitability which is thought to be consistent with an improved ability of the neuromuscular system to recruit the affected musculature. That is, a stronger response is evoked by a given neural signal. ${ }^{32}$ Our results are therefore consistent with the suggestion that the gluteal activation warm-up increased the corticomotor excitability of the glutes acutely. Such a phenomenon would offer an exciting validation of the use of therapeutic exercises to prime performance.

Of course, it should also be acknowledged that these suggestions are based on an entirely credulous interpretation of our findings and that both the muscle force estimates and the EMG data should be treated with caution. A further explanation for the discrepancy between EMG measurements and muscle force estimates might simply be that the muscle force estimates are incorrect. However, this alternative explanation still would not explain why the ground reaction forces remained unchanged when the EMG activity was decreased.

\section{Musculoskeletal models can provide clinical insight of relevance to practitioners}

In this study, the use of FreeBody was a key aspect of the experimental approach. Musculoskeletal modellers envisage that such models can be used to evaluate and simulate movement to provide general advice for clinicians, but that ultimately such models will progress to a point where they can be used on a subject-specific basis to guide medical, surgical, therapeutic and exercise interventions. ${ }^{33} 34$ This study represents an important milestone towards this goal as, to our knowledge, this is the first study to employ a musculoskeletal model to evaluate the acute effect of an exercise intervention. The results of this study exemplify how musculoskeletal models can provide insight that may not be available from more traditional approaches. In particular, in this study, the EMG results alone might indicate that the gluteal activation warm-up actually caused a decrease in the involvement of the glutes in the movement, when the model analysis suggests the contrary.

Of course, the results of musculoskeletal modelling studies like this one are not without their own caveats. In particular, it is important that readers understand that the muscle forces reported here are estimates and are not directly measured. Similarly, the model employed here is generic and including further subject specific detail is likely to improve the accuracy of the muscle force estimation. ${ }^{23} 35$

\section{Conclusions}

The results of this study provide support for the employment of gluteal activation exercises as a strategy to acutely facilitate the recruitment of the gluteal and hamstring musculature and that this may result in improved movement quality. In addition, the results of this study add tacit support to the notion that the mechanism of the increased recruitment is through a potentiation of the neuromuscular system such that a given neural drive evokes greater force production.

Acknowledgements We would like to thank Jon E Goodwin and Jack Lineham for their advice and support in the planning and execution of this study.

Contributors MP and DJC conceived the study. MP collected the data used in the study. All authors were involved in the study design, analysis and interpretation of the data, and preparation of the manuscript. All authors approved the final version and agreed to be accountable for the work.

Competing interests None declared.

Ethics approval St Mary's University Ethical Review Board.

Provenance and peer review Not commissioned; internally peer reviewed.

Open Access This is an Open Access article distributed in accordance with the Creative Commons Attribution Non Commercial (CC BY-NC 4.0) license, which permits others to distribute, remix, adapt, build upon this work noncommercially, and license their derivative works on different terms, provided the original work is properly cited and the use is non-commercial. See: http:// creativecommons.org/licenses/by-nc/4.0/

Correction notice This paper has been amended since it was published Online First. Owing to a scripting error, some of the publisher names in the references were replaced with 'BMJ Publishing Group'. This only affected the full text version, not the PDF. We have since corrected these errors and the correct publishers have been inserted into the references.

(c) Article author(s) (or their employer(s) unless otherwise stated in the text of the article) 2017. All rights reserved. No commercial use is permitted unless otherwise expressly granted.

\section{REFERENCES}

1. Jeffreys I. Warm up revisited - the "ramp"method of optimising performance preparation. Uksca J 2006;6:15-19.

2. Nagano A, Komura T, Fukashiro S, et al. Force, work and power output of lower limb muscles during human maximal-effort countermovement jumping. J Electromyogr Kinesiol 2005;15:367-76.

3. Mero A, Komi PV, Gregor RJ. Biomechanics of Sprint running. A review. Sports Medicine 1992;13:376-92.

4. Kyröläinen $\mathrm{H}$, Avela J, Komi PV. Changes in muscle activity with increasing running speed. J Sports Sci 2005;23:1101-9.

5. Nelson-Wong E, Gregory DE, Winter DA, et al. Gluteus medius muscle activation patterns as a predictor of low back pain during standing. Clin Biomech 2008;23:545-53.

6. Kankaanpää M, Taimela S, Laaksonen D, et al. Back and hip extensor fatigability in chronic low back pain patients and controls. Arch Phys Med Rehabil 1998;79:412-7.

7. Robinson RL, Nee RJ. Analysis of hip strength in females seeking physical therapy treatment for unilateral patellofemoral pain syndrome. J Orthop Sports Phys Ther 2007;37:232-8.

8. Ireland ML, Willson JD, Ballantyne BT, et al. Hip strength in females with and without patellofemoral pain. J Orthop Sports Phys Ther 2003;33:671-6.

9. Distefano LJ, Blackburn JT, Marshall SW, et al. Gluteal muscle activation during common therapeutic exercises. J Orthop Sports Phys Ther 2009;39:532-40.

10. Barton CJ, Lack S, Malliaras $P$, et al. Gluteal muscle activity and patellofemoral pain syndrome: a systematic review. Br J Sports Med 2013;47:207-14. 
11. Crow JF, Buttifant D, Kearny SG, et al. Low load exercises targeting the gluteal muscle group acutely enhance explosive power output in elite athletes. J Strength Cond Res 2012;26:438-42.

12. Comyns T, Kenny I, Scales G. Effects of a low-load gluteal warm-up on explosive jump performance. J Hum Kinet 2015;46:177-87.

13. Barry L, Kenny I, Comyns T. Performance effects of repetition specific gluteal activation protocols on acceleration in male rugby union players. J Hum Kinet 2016;54:33-42.

14. Healy R, Harrison AJ. The effects of a unilateral gluteal activation protocol on single leg drop jump performance. Sports Biomech 2014;13:33-46.

15. Cochrane DJ, Harnett MC, Pinfold SC. Does short-term gluteal activation enhance muscle performance? Res Sports Med 2017;25:156-65.

16. Cleather DJ, Bull AM. The development of a segment-based musculoskeletal model of the lower limb: introducing FreeBody. $R$ Soc Open Sci 2015;2:140449.

17. Montgomery J, Hislop H, Connelly B, et al. Daniel's and Worthingham's muscle testing: techniques of manual examination. Saunders, 2007.

18. Cleather DI, Bull AM. Lower-extremity musculoskeletal geometry affects the calculation of patellofemoral forces in vertical jumping and weightlifting. Proc Inst Mech Eng H 2010;224:1073-83.

19. Cleather DJ, Bull AM. An optimization-based simultaneous approach to the determination of muscular, ligamentous, and joint contact forces provides insight into musculoligamentous interaction. Ann Biomed Eng 2011;39:1925-34.

20. Cleather DJ, Bull AM. Knee and hip joint forces - sensitivity to the degrees of freedom classification at the knee. Proc Inst Mech Eng $\mathrm{H}$ 2011;225:621-6.

21. Cleather DJ, Goodwin JE, Bull AM. An optimization approach to inverse dynamics provides insight as to the function of the biarticular muscles during vertical jumping. Ann Biomed Eng 2011;39:147-60.

22. Cleather DJ, Goodwin JE, Bull AMJ. Erratum to: an optimization approach to Inverse Dynamics provides Insight as to the function of the biarticular muscles during vertical jumping. Ann Biomed Eng 2011;39:2476-8

23. Ding Z, Nolte D, Kit Tsang C, et al. In vivo knee contact force prediction using patient-specific musculoskeletal geometry in a segment-based computational model. J Biomech Eng 2016;138:021018.

24. Price PDB, Gissane C, Cleather DJ. The evaluation of the FreeBody lower limb model during activities of daily living. 2016.

25. Klein Horsman MD, Koopman HF, van der Helm FC, et al. Morphological muscle and joint parameters for musculoskeletal modelling of the lower extremity. Clin Biomech 2007;22:239-47.

26. Dumas R, Aissaoui R, de Guise JA. A 3D generic inverse dynamic method using wrench notation and quaternion algebra. Comput Methods Biomech Biomed Engin 2004;7:159-66.

27. de Leva P. Adjustments to Zatsiorsky-Seluyanov's segment inertia parameters. J Biomech 1996;29:1223-30.

28. Crowninshield RD, Brand RA. A physiologically based criterion of muscle force prediction in locomotion. J Biomech 1981;14:793-801.

29. Raikova RT. Investigation of the influence of the elbow joint reaction on the predicted muscle forces using different optimization functions. J Musculoskelet Res 2009;12:31-43.

30. Konrad P. The ABC of EMG: a practical introduction to kinesiological electromyography . 1, 2005:30-5.

31. Muthuraman M, Govindan RB, Deuschl G, et al. Differentiating phase shift and delay in narrow band coherent signals. Clin Neurophysiol 2008;119:1062-70.

32. Fisher BE, Southam AC, Kuo YL, et al. Evidence of altered corticomotor excitability following targeted activation of gluteus maximus training in healthy individuals. Neuroreport 2016;27:415-21.

33. Cleather DJ, Bull AM. The development of lower limb musculoskeletal models with clinical relevance is dependent upon the fidelity of the mathematical description of the lower limb. Part I: equations of motion. Proc Inst Mech Eng H 2012;226:120-32.

34. Cleather DJ, Bull AM. The development of lower limb musculoskeletal models with clinical relevance is dependent upon the fidelity of the mathematical description of the lower limb. Part 2: patient-specific geometry. Proc Inst Mech Eng $\mathrm{H}$ 2012;226:133-45.

35. Southgate DF, Cleather DJ, Weinert-Aplin RA, et al. The sensitivity of a lower limb model to axial rotation offsets and muscle bounds at the knee. Proc Inst Mech Eng H 2012;226:660-9. 